\title{
CUBIC SUBFIELDS OF EXCEPTIONAL SIMPLE JORDAN ALGEBRAS
}

\author{
H. P. PETERSSON AND M. L. RACINE ${ }^{l}$
}

\begin{abstract}
Let $E / k$ be a cubic field extension and $J$ a simple exceptional Jordan algebra of degree 3 over $k$. Then $E$ is a reducing field of $J$ if and only if $E$ is isomorphic to a (maximal) subfield of some isotope of $J$. If $k$ has characteristic not 2 or 3 and contains the third roots of unity then every simple exceptional Jordan division algebra of degree 3 over $k$ contains a cyclic cubic subfield.
\end{abstract}

Exceptional Jordan division algebras were first constructed by Albert [2, 3]. Tits gave two constructions which yield all finite-dimensional exceptional simple Jordan algebras over fields of characteristic not 2 [4]. Then McCrimmon [5, 6] gave a particularly elegant characteristic free formulation of these results. Recently Zelmanov [14] proved that any exceptional Jordan division algebra of characteristic not 2 is finite dimensional over its center. Finally, the two Tits constructions were treated in a unified fashion via the Tits process introduced in [8].

There remains the isomorphism problem. If the algebra is not a division algebra, by results of Albert, Jacobson and Springer, isomorphism is equivalent to the isometry of quadratic forms in 8 and 24 variables (see [11] for a characteristic free version). In the case of division algebras the answer is not known and the results of this note represent small steps in the direction of a solution. We recall a few useful results and definitions. Let $k$ be a field. By a cubic form with adjoint and base point over $k$ we mean a triple $(N, \#, 1)$ consisting of a cubic form $N$ on some $k$ vector

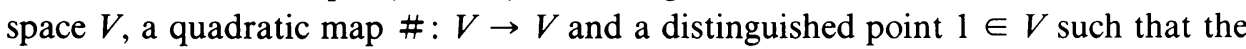
identities

$$
\begin{gathered}
v^{\# \#}=N(v) v, \\
N(1)=1, \\
T\left(v^{\#}, w\right)=(D N)_{v}(w), \\
1^{\#}=1, \\
1 \times v=T(v) 1-v
\end{gathered}
$$

hold under all scalar extensions. Here " $D$ " indicates the total differential of a rational map, $T=-\left(D^{2} \log N\right)$, is the second logarithmic derivative of $N$ evaluated at $1, T(v)=T(v, 1)$, and $\times$ is the bilinearization of the adjoint \#. We call $T$ the

Received by the editors May 26, 1983.

1980 Mathematics Subject Classification. Primary 17C40.

'The research of the second author was supported in part by an NSERC grant and by a von Humboldt Fellowship at the University of Münster. 
trace form associated with $(N, \#, 1)$. McCrimmon [5] has shown that the base point 1 and the $U$-operator defined by

$$
U_{v} w=T(v, w) v-v^{\#} \times w
$$

give $V$ the structure of a unital quadratic Jordan algebra over $k$, written as $g=g(N, \#, 1) \cdot g$ is of degree 3 over $k$.

$$
\begin{gathered}
v^{3}-T(v) v^{2}+T\left(v^{\#}\right) v-N(v) 1=0, \\
v^{\#}=v^{2}-T(v) v+T\left(v^{\#}\right) 1 .
\end{gathered}
$$

We refer to the quadratic form $S(x)=T\left(x^{\#}\right)$ as the quadratic trace.

If $f(N, \#, 1)$ is exceptional simple of degree 3 over $k$ (one would like to say "central simple over $k$ " but the usual notion of center is not a natural one in the quadratic setting [6]) then $g$ is a Jordan division algebra or is isomorphic to $H_{3}(C, g), C$ an octonion algebra, $g=\operatorname{diag}\left(g_{1}, g_{2}, g_{3}\right) \in \mathrm{GL}_{3}(k)$. A nonzero idempotent $e$ is absolutely primitive if every element of $U_{e} g$ has the form $\alpha e+z$ where $\alpha \in k$ and $z$ is nilpotent. A unital Jordan algebra is said to be reduced if $1=\sum_{i=1}^{n} e_{i}$, where the $e_{i}$ are absolutely primitive idempotents. The algebra $H_{3}(C, g)$ is reduced. We say that $H_{3}(C, g)$ is split when $C$ is split. Writing $\alpha[i i]=\alpha e_{i i}, \alpha \in k$, $1 \leqslant i \leqslant 3$ and $a[i j]=g_{i} a e_{i j}+g_{j} \bar{a} e_{j i}, a \in C, 1 \leqslant i, j \leqslant 3$, where the $e_{i i}, e_{i j}$ are the ordinary matrix units, then arbitrary elements of $q$ have the form

$$
x=\sum \xi_{i}[i i]+\sum_{(123)} x_{i}[j k], \quad y=\sum \eta_{i}[i i]+\sum_{(123)} y_{i}[j k],
$$

where $\sum_{(123)}$ denotes the sum over all cyclic permutations of (123).Then

$$
\begin{aligned}
& T(x, y)=\sum \xi_{i} \eta_{i}+\sum_{(123)} g_{i} g_{j} t\left(x_{i}, y_{j}\right) \text {, } \\
& x^{\#}=\sum_{(123)}\left\{\xi_{i} \xi_{j}-g_{i} g_{j} n\left(x_{k}\right)\right\}[k k]+\sum_{(123)}\left\{g_{i}\left(\overline{x_{j} x_{k}}\right)-\xi_{i} x_{i}\right\}[j k] .
\end{aligned}
$$

Of course $-, n, t$ are the canonical involution, norm form and linearization of $n$ of C.

We say that a simple algebra $\mathcal{G}$ is absolutely simple over $k$ if it remains simple under all scalar extensions (see [6] for an example of a simple nonabsolutely simple algebra).

The following proposition can be proved using structure theory (cf. [4]) but can also be easily obtained using the methods of [9].

Proposition 1. Let $q$ be a Jordan division algebra of degree 3 generated by two elements. Then $q$ has dimension 3 or 9 . Every nine-dimensional Jordan division algebra of degree 3 is isomorphic to precisely one of the following:

(a) $D^{+}$for some central associative division algebra $D$ of degree 3 over $k$,

(b) $H(D, *)$ for some central associative division algebra $\left(D,{ }^{*}\right)$ of degree 3 with involution of the second kind over $k$,

(c) a purely inseparable field extension of exponent 1 over $k$, where $k$ has characteristic 3 . 
We will also need

SPRINGER'S THEOREM [12]. Let $q$ be an anisotropic quadratic form over $k$ and $E / k$ an algebraic field extension of odd degree. Then the scalar extension of $q$ to $E$ is an anisotropic quadratic form over $E$.

As far as we know, the proof of this result is always given over fields of characteristic not 2 . However, adding a few simple adjustments, it is not hard to make it work in general.

The complexity of the structure of an exceptional Jordan division algebra depends to a large extent on the variety of its nine-dimensional subalgebras. These in turn are all obtainable from separable cubic subfields via the Tits constructions [9]. It is therefore natural to ask for conditions characterizing those cubic extensions of the base field that live inside a given exceptional simple Jordan algebra.

There is a classical result along these lines in the setting of associative algebras (cf. Albert [1, IV Theorem 27, p. 61]): A finite algebraic extension $K / k$ is a maximal subfield of a central simple associative $k$-algebra $A$ of the same degree if and only if $K$ is a splitting field of $A$. In the setting of Jordan algebras, and in this context, the property of being reduced appears to be more natural than the one of being split. Thus the question presents itself whether or not a cubic extension $E / k$ is a maximal subfield of a simple exceptional Jordan algebra $q$ of degree 3 over $k$ if and only if $E$ reduces $g$.

It is not hard to answer this question in the negative: Let $C$ be the (unique) octonion division algebra over the field $\mathbf{Q}$ of rational numbers and put $g=H_{3}(C)$. Then $E=\mathbf{Q}(\xi)$, where $\xi$ is a root of the polynomial $f=x^{3}+x+1 \in \mathbf{Q}[x]$, reduces $q$ since $g$ itself is reduced to begin with. On the other hand, the assumption that $E$ is a subfield of $\mathcal{G}$ implies that $E \otimes_{\mathbf{Q}} \mathbf{R}$ is a subalgebra of $\mathscr{f} \otimes_{\mathbf{Q}} \mathbf{R}$. But the Jordan algebra $f \otimes_{\mathbf{Q}} \mathbf{R}$ is formally real whereas $E \otimes_{\mathbf{Q}} \mathbf{R}$, since $f$ has only one real root, is isomorphic to $\mathbf{R} \otimes \mathbf{C}$, and $\mathbf{C}$ is not formally real, so we have arrived at a contradiction.

The conditioned reflex to save this kind of game in the theory of Jordan algebras consists in shifting emphasis from $g$ to appropriate isotopes. This strategy turns out to be successful in the case under consideration, for we can prove

THEOREM 2. Let $E / k$ be a cubic field extension and $q$ a simple exceptional Jordan algebra of degree 3 over $k$. Then $E$ is a reducing field of $g$ if and only if $E$ is isomorphic to a (maximal) subfield of some isotope of $\mathcal{G}$.

Proof. If $E$ is a maximal subfield of some isotope of $\mathcal{G}$, it reduces that isotope, hence $q$. Conversely, suppose $E$ is a reducing field of $q$. Given a field extension $F / k$, let us agree to call $E$ a reducing field of $F$ in case $F \otimes E$ has zero divisors. We first contend that $g$ contains a nine-dimensional subalgebra $\mathcal{G}^{\prime}$ of degree 3 which is absolutely simple or a division algebra, and is reduced by $E$. If $\mathscr{g}$ is reduced, any reduced nine-dimensional absolutely simple subalgebra of degree 3 will do. If $q$ is a division algebra, we write $E=k(\theta)$ and, as $g_{E}$ is not a division algebra, we may find elements $v_{0}, v_{1}, v_{2} \in \mathcal{G}$, not all zero, such that $v=v_{0}+\theta v_{1}+\theta^{2} v_{2} \in \mathcal{G}_{E}$ is not 
invertible. For $v_{0} \neq 0$, we pass to the isotope $g^{(y)}, y=v_{0}^{-1}$, and then have $v_{0}=1^{(y)}$. Hence there is no harm in assuming that $v_{0}$ is either 0 or 1 . It follows from Proposition 1 that $v_{0}, v_{1}, v_{2}$ all belong to a common subalgebra $g^{\prime}$ of $q$ having dimension 9. Thus $v$ lives as a noninvertible element in $g_{E}^{\prime}$, and $E$ reduces $\mathcal{g}^{\prime}$. Our intermediate contention is thereby proved. Theorem 1 of [11] and Proposition 1 now leave the following options for $g^{\prime}$.

Case $1 . g^{\prime} \cong A^{+}, A$ a central simple associative algebra of degree 3 over $k$. Then $E$, reducing $A^{+}$, splits $A$ and thus, by [1, IV Theorem 27, p. 61] is isomorphic with a maximal subfield of $A$, hence of $\mathcal{G}$.

Case $2 . g^{\prime} \cong H\left(B,{ }^{*}\right),(B, *)$ a central simple associative algebra of degree 3 with involution of the second kind over $k$. Writing $K$ for the center of $B$, which we may assume to be a separable quadratic field extension of $k$ (since Case 1 already has been taken care of), $g_{K}^{\prime}$ becomes isomorphic with $B^{+}$and is reduced by $E_{K}$. Therefore, arguing as in Case 1, we may assume $E \subset E_{K} \subset B^{+}$. Now $E_{K} / K$ is still generated by the element $\theta$, whose minimum polynomial over $K$ of course has coefficients in $k$. Hence it follows from a theorem of Albert [1, X Theorem 15, p. 157] that there exists a $K / k$-involution ${ }^{* \prime}$ on $B$ satisfying $\theta^{* \prime}=\theta$ i.e., $E \subset H\left(B,{ }^{* \prime}\right)$. Since we are dealing with involutions of second kind, ${ }^{* \prime}$ differs from ${ }^{*}$ by an inner automorphism that arises from a ${ }^{*}$-symmetric element $y \in B^{\times}$. Therefore, $H\left(B,{ }^{* \prime}\right)$ being isomorphic with the isotope $g^{\prime(y)}, E$ is isomorphic with a subfield of $g^{\prime(y)}$, hence of $g^{(y)}$.

Case $3 . g^{\prime} \cong F^{+}, F / k$ a purely inseparable field extension of exponent 1 . Choosing an intermediate extension $F^{\prime} / k$ of degree 3 , generated by an element $\theta^{\prime} \in F-k$, we first assume that $E$ reduces $F^{\prime}$. Then the minimum polynomial of $\theta^{\prime}$ over $k$ becomes reducible over (and therefore has a root in) $E$, whence $E$ and $F^{\prime}$ are isomorphic over $k$. On the other hand, if $E$ does not reduce $F^{\prime}$, the same argument applies to $E \otimes F^{\prime}$ and $F$ over $F^{\prime}$, in place of $E$ and $F^{\prime}$ over $k$. In any event, $E$ turns out to be isomorphic to a subfield of $F^{+} \cong \mathcal{g}^{\prime}$, hence to a subfield of $\mathscr{q}$.

Let $q$ be a simple exceptional Jordan algebra of degree 3 over $k$. If it contains a subalgebra $A^{+}, A$ central simple associative of degree 3 over $k$, then as a vector space $\mathcal{G}=A \oplus A \oplus A$ and the Jordan structure is obtained by applying the first Tits construction. Combining Theorem 2 with Corollaries 4.2 and 4.9 of [10], we obtain

COROLlaRY 3. Let $E / k$ be a cubic field extension and $q$ a simple exceptional Jordan algebra of degree 3 over $k$ arising from the first Tits construction. Then $E$ is a splitting field of $\mathcal{G}$ if and only if $E$ is isomorphic to a subfield of $q$.

Probably the most important problem in this context has been raised by Albert [4], reîerred to in what follows as the Albert Problem: Does every central exceptional Jordan divisor algebra contain a cyclic cubic subfield? There are, aside from the striking analogy to Wedderburn's Theorem on the cyclicity of central associative division algebras of degree 3, various good reasons for heing interested in this question, which remains unsolved to this day. For example, Springer's theory of twisted composition algebras [13] becomes applicable to exceptional simple Jordan algebras only in the presence of cyclic cubic subfields. We can give an affirmative answer to the Albert problem when some restrictions are imposed on the base field. 
THEOREM 4. Suppose $k$ has characteristic not 2, 3 and contains the third roots of unity. Then every exceptional Jordan division algebra of degree 3 over $k$ contains $a$ cyclic cubic subfield.

Proof. Our hypotheses imply that a cubic field extension of $k$ is cyclic if and only if it is pure. Hence, writing $S$ for the quadratic trace of a given exceptional Jordan division algebra $q$ of degree 3 over $k$, and invoking (7), it suffices to show that the quadratic form $S$ is isotropic on the elements of trace zero in $g$. Applying Springer's Theorem to any cubic subfield $E$ of $\mathscr{f}$, it is actually enough to establish isotropy of $S^{\prime}=S \otimes E$ on the elements of trace zero in $g^{\prime}=g \otimes E$. Now $g^{\prime}$ is reduced and so has the form $g^{\prime}=H_{3}(C, g)$, for some octonion algebra $C$ over $E$ and some diagonal matrix $g \in \mathrm{GL}_{3}(E)$. Write $V$ for the subspace of $g^{\prime}$ spanned over $E$ by the elements $b_{i}=1[i i]-1[33]$ for $i=1,2$, which consists entirely of elements of trace zero. Consulting (9) and (10) it is easily checked that, relative to the basis $b_{1}, b_{2}$ of $V$, the symmetric bilinear form corresponding to the restriction of $S^{\prime}$ to $V$ has the matrix

$$
\left[\begin{array}{ll}
-2 & -1 \\
-1 & -2
\end{array}\right]
$$

whose determinant is 3 and hence, as -3 is a square in $k$, $\equiv-1 \bmod E^{\times 2}$. Thus $S^{\prime}$ must be hyperbolic on $V$, and our contention follows.

In spite of the evidence in favour of an affirmative answer produced by Theorem 4 and Wedderburn's result on the cyclicity of associative division algebras of degree 3 , there is also evidence to the contrary, based on properties of nine-dimensional Jordan division algebras of the second rather than the first kind, which conceivably are more typical for what may happen in the exceptional case. The properties we have in mind may be found in the following proposition.

Proposition 5. The iterated Laurent series field $k=\mathbf{R}\left(\left(X_{1}, \ldots, X_{n}\right)\right)$ in $n \geqslant 1$ variables $X_{1}, \ldots, X_{n}$ over the field $\mathbf{R}$ of real numbers does not admit any cyclic cubic field extensions. On the other hand, for $n \geqslant 2$ there exist unramified cubic extensions of $k$ (viewed canonically as a local field). If $E$ is such and $\pi$ is a prime element of $k$, the first Tits construction $\mathcal{G}(E, \pi)$ is a nine-dimensional Jordan division algebra of the second kind all of whose cubic subfields are separable noncyclic.

Proof. The arguments produced in [7, pp. 19-20] establish the first of the above assertions. The rest follows from standard properties of local fields.

Unfortunately, all central simple exceptional Jordan algebras over $\mathbf{R}\left(\left(X_{1}, \ldots, X_{n}\right)\right)$ are reduced [7, Theorem 7], so, in order to find a negative answer to the Albert Problem, one has to look for other fields.

\section{REFERENCES}

1. A. A. Albert, Structure of algebras, Amer. Math. Soc. Colloq. Publ., Vol. 24, Amer. Math. Soc., Providence, R. I., 1939.

2. __ A construction of exceptional Jordan division algebras, Ann. of Math. (2) 67 (1958), 1-28.

3. __ On exceptional Jordan division algebras, Pacific J. Math., 15 (1965), 377-404.

4. N. Jacobson, Structure and representations of Jordan algebras, Amer. Math. Soc. Colloq. Publ., Vol. 39, Amer. Math. Soc., Providence, R. I., 1968.

5. K. McCrimmon, The Freudenthal-Springer-Tits constructions of exceptional Jordan algebras, Trans. Amer. Math. Soc. 139 (1969), 495-510. 
6. __ The Freudenthal-Springer-Tits constructions revisited, Trans. Amer. Math. Soc., 148 (1970), 293-314

7. H. P. Petersson, Exceptional Jordan division algebras over a field with a discrete valuation, J. Reine Angew. Math., 274 (1975), 1-20.

8. H. P. Petersson and M. L. Racine, Jordan algebras of degree 3 and the Tits process, J. Algebra (to appear).

9. __ Classification of algebras arising from the Tits process, J. Algebra (to appear).

10. Springer forms and the first Tits construction of exceptional Jordan division algebras, Manuscripta Math.

11. M. L. Racine, A note on quadratic Jordan algebras of degree 3, Trans. Amer. Math. Soc. 164 (1972), 93-103.

12. T. A. Springer, Sur les formes quadratiques d'indice zéro, C. R. Acad. Sci. Paris 234 (1952), 1517-1519.

13. __ Oktaven, Jordan-Algebren und Ausnahmegruppen, Univ. Göttingen Lecture Notes, Göttingen, 1963.

14. E. I. Zelmanov, Jordan division algebras, Algebra i Logika 18 (1979), 286-310.

FACHBEREICH MATHEMATIK UND INFORMATIK, FERN UNIVERSiTÄt, LÜTZOWSTRASSE 125, D - 58 HageN, BundesRepublik Deutschland, Federal Republic of Germany

Department of Mathematics, University of Ottawa, OtTaWa, Ontario K1N 9B4, Canada 